\title{
Equating tasks and sustaining attention in children and adults: The methodological and theoretical utility of $d^{\prime}$ matching
}

\author{
CYNTHIA LAURIE-ROSE, LAURA BENNETT-MURPHY, LORI M. CURTINDALE, \\ ANDREA L. GRANGER, and HEIDI B. WALKER \\ Otterbein College, Westerville, Ohio
}

\begin{abstract}
The present study develops a method to create task equivalency for children and adults in vigilance research. Experiment 1 employed the signal detection index of perceptual sensitivity $\left(d^{\prime}\right)$ to calibrate vigilance tasks for 32 children (ages 7-8) and 32 adults. The results of Experiment 1 suggest that task equivalency across groups can be attained, and a vigilance decrement was observed for children. In Experiment 2 , the task parameter of event rate was manipulated across two vigils. Again, 32 children (ages 7-8) and 32 adults participated. The results suggest that a reverse event rate effect emerged for both children and adults. In addition, developmental differences in response to the event rate manipulation and with respect to the vigilance decrement emerged: Children benefited less from the manipulation and were more likely to demonstrate a vigilance decrement than were adults. No developmental differences emerged for the sensitivity decrement. The results are explained with reference to how task demands interact with participant arousal, and implications for development and for future research are discussed.
\end{abstract}

Vigilance or sustained attention describes the ability of individuals to remain focused in attention and alert to stimuli for extended periods of time. The psychophysics of vigilance in adults has been thoroughly investigated and documented over the last 50 years (cf. Warm \& Dember, 1998; Warm \& Jerison, 1984). Recently, the study of attention in children has, as Cooley and Morris (1990) state, "paralleled interest in defining and measuring the attentional construct in normal adults" (p. 240). Whether one examines social interactions (Goldsmith \& Rogoff, 1997), cognitive development (cf. Bell, 1998), or the early identification of children at risk (cf. Bornstein, 1990), attentional processes are central to explanations of behavior.

Yet over the years, significant criticism has been leveled at the methodological limits of childhood vigilance studies (e.g., Berch \& Kanter, 1984; Corkum \& Siegel, 1993; Halperin, Sharma, Greenblatt, \& Schwartz, 1991; Losier, McGrath, \& Klein, 1996; McKay, Halperin, Schwartz, \& Sharma, 1994; Seidel \& Joschko, 1990; Trommer, Hoeppner, Lorber, \& Armstrong, 1988). Studies of children have, for example, been limited by samples suffering from small

C. L.-R. acknowledges Otterbein College for a sabbatical leave and research funds supporting this study. The authors thank Ed Zettler of the Columbus Diocese and George Tombaugh of Westerville Schools for providing access to children. The authors also thank Christine Abraham, Mandi Jackson, Katie Nikzad, and Liz Stimer for their assistance in running early pilot subjects. L.M.C. is now in the Department of Psychology at Bowling Green State University. Correspondence should be sent to C. Laurie-Rose at Department of Psychology, One Otterbein College, Westerville, OH 43081 (e-mail: claurie-rose@otterbein.edu). group size, comorbid diagnosis, or heterogeneity of comparison groups in terms of gender, intelligence, and age. Task-related problems also have arisen, with many studies neglecting to account for factors known to influence adult performance, such as stimulus duration, event rate, signal probability, and type of task. Furthermore, task complexity and length of task have remained largely uncontrolled (Seidel \& Joschko, 1990).

Several of the above concerns derive from the fact that child research has proceeded in relative isolation from the vast adult literature. For example, few studies have utilized adult comparison groups. A serious impediment to examining jointly the performance of children, adolescents, and adults has been the difficulty of designing comparable tasks across age. Adult tasks yield basal effects for children, and tasks developed for children prove inappropriately easy for adults. The consequent ceiling effects limit conclusions regarding development (Halperin et al., 1991). Such problems undermine the formation of developmental models of attention, for age group differences might reflect the different tasks used with adults and children rather than reveal signs of developmental change.

Many researchers concerned with developmental trends in perception and cognition have noted the critical importance of devising tasks specifically suited for testing across age groups (Berch \& Kanter, 1984; Enns, Brodeur, \& Trick, 1998; Giambra \& Quilter, 1988; Halperin, 1996; Tomporowski \& Tinsley, 1996). Indeed, some investigators point to limits in their own experiments due to the uneven influence of task difficulty across age (cf. McKay et al., 1994). The present study tests a method for equat- 
ing vigilance tasks across age groups by employing $d^{\prime}$, the signal detection index of perceptual sensitivity, to determine task equivalency.

The combination of familiarity, reliability, and precedent led us to choose the $d^{\prime}$ measure. Although proportion correct is a familiar index, it is not unbiased. Likewise, although the sensitivity measure $\mathrm{p}(\mathrm{c}) \max$ is unbiased (Macmillan \& Creelman, 1991), it does not have the familiarity in the vigilance field of the more commonly used $d^{\prime}$, which has long been integral to the study of vigilance (Warm \& Jerison, 1984). Methodologically, $d^{\prime}$ allows reliable comparisons across different experiments and across different values of physical variables (Macmillan \& Creelman, 1991). Importantly, recent evidence (See, Howe, Warm, \& Dember, 1995) has lain to rest questions (cf. Teichner, 1974) as to whether vigilance data meet the statistical assumptions necessary to perform signal detection analyses. Finally, precedent exists for using $d^{\prime}$ as a means to calibrate tasks (Davies, Jones, \& Taylor, 1984). Studies have sought to match difficulty across tasks by equating group (Hatfield \& Loeb, 1968) or individual (e.g., Tyler, Waag, \& Halcomb, 1972) values of $d^{\prime}$. For example, Parasuraman and Mouloua (1987) equated simultaneous and successive discrimination vigilance tasks in adults by applying the perceptual sensitivity index of $d^{\prime}$. In another experiment, Parasuraman, Nestor, and Greenwood (1989) used $d^{\prime}$ to assure task comparability in young and older adults. Given all the advantages of $d^{\prime}$, the present study developed procedures necessary to achieve $d^{\prime}$ matching of signal detectability in children and adults.

We see the creation of comparable tasks as significant for building developmental models of attention. As Enns et al. (1998) have described, theories of attention have been largely silent on developmental issues. Disparate fields, however - the adult vigilance literature and the extensive clinical child literature, with notable contributions from the area of clinical neuropsychology (cf. Cooley \& Morris, 1990) — can provide a framework for building such models in children.

The adult literature widely applies two theories of vigilance performance: resource allocation theory, derived from general information processing models, and arousal theory. Resource allocation theory suggests that capacity limitations exist in information processing and that various task parameters may differentially exhaust available resources. Arousal theory suggests that the inherently boring nature of a sustained attention task reduces an observer's physiological and behavioral excitation to suboptimal levels and thus hinders performance (Parasuraman, 1984). Recently, Matthews and Davies (1998a) integrated the resource allocation and arousal models to overcome limitations posed by arousal theory alone (cf. Hockey, 1984). They suggested that in a condition of heightened arousal - in this case, of sufficiently high task demand-individuals mobilize greater resources.

In the clinical childhood literature, researchers have successfully applied optimal stimulation theory to explain the influence of arousal in children. This theory, rooted in homeostatic models, claims that organisms strive toward optimal levels of stimulation (cf. Berlyne, 1960). Insufficient stimulation or excessive stimulation will lead to impaired performance. Zentall and Zentall (1983) used optimal stimulation theory to explain behavior in children diagnosed with autism and in other children diagnosed with attention deficit disorder, who are often respectively described as underaroused and overaroused.

As do resource allocation models, optimal stimulation theories give strong consideration to the influences of task demand and developmental stage. Consensus exists among researchers that experimental factors such as task type, task complexity, practice, rate and duration of stimuli, and type of responses will differentially affect performance (Leung, Leung, \& Tang, 2000; Zentall \& Zentall, 1983). Van der Meere and colleagues (van der Meere \& Stemerdink, 1999; van der Meere, Vreeling, \& Sergeant, 1992) have shown that certain task demands can raise a child's energy pool, a conclusion similar to that of Matthews and Davies (1998a) with adults. Zentall and Zentall suggested that younger children may be more vulnerable to variations in the level of stimulation because they have less experience with cognitive modulation than do older children and adults. Furthermore, Zentall and Zentall speculated that adults tolerate a wider range of stimulation than do children. Children's narrowed range makes them vulnerable even to subtle manipulations in the task. In its incorporation of experimental and developmental factors, optimal stimulation theory provides a link between the arousal and resource allocation models. Furthermore, as van der Meere and colleagues have indicated, the theory can be applied to the study of typical as well as of atypical attention.

The present study, by examining children and adults under conditions of both high and low event rates, offers a method for comparing typical attention in children and adults. Researchers in vigilance often have been challenged by variability in childhood patterns of sustained attention. Some have described the period between ages 6 and 8 as relatively stable (Levy, 1980), with rapid changes in sustained attention from ages 8 to 10 (Davies et al., 1984; Rebok et al., 1997; Sykes, Douglas, Weiss, \& Minde, 1971). Greenberg and Waldman (1993), however, have described rapid improvement of attention throughout ages 6 to 12, with decelerated growth in late childhood. McKay et al. (1994), in contrast, reported relative stability between the ages of 7 and 11, with more pronounced changes from age 11 to adulthood. Such disagreement is a function of differences in method, task, and analysis.

For the present study, we recruited 7- and 8-year-old children. The finding that the relationship between sustained attention and school success is quite strong in the elementary years (NICHD Early Child Care Research Network, 2003) provided a compelling reason to choose children from the elementary population. As indicated above, moderate consensus exists that children of 7 and 8 years show relative stability in the development of sustained attention among school children (Levy, 1980; McKay et al., 1994). Because children younger than 7 and 
older than 8 experience more rapid shifts in attentional capacities, the selected age group seemed most desirable (Davies et al., 1984; Rebok et al., 1997; Sykes et al., 1971). Such stability reduces variations within groups, allowing for more sensitive comparisons between groups. Finally, because children with ADHD most often are diagnosed between the ages of 7 and 9, the peak period of referral (DeWolfe, Byrne, \& Bawden, 1999), studies of typically developing children within that age range can provide a basis for comparison with the extensive clinical literature. For all of the above reasons, the selected age group seemed best suited for applying our methodology to an understanding of the development of typical attention.

\section{EXPERIMENT 1}

Initially, we attempted to follow the lead of Parasuraman and Mouloua (1987) and match under brief, alerted conditions. Extensive piloting, however, revealed that a short watch did not provide replicable data: Great individual variations became evident, especially with children, suggesting that participants acclimated to the task at different rates. Hatfield and Soderquist (1970), on the other hand, described $d^{\prime}$ matching procedures under vigilant rather than alerted conditions. We experimented, therefore, with obtaining $d^{\prime}$ values for participants in a continuous 14-min vigil that was divided into seven 2min periods of watch. This 14-min vigil was preceded by a 2-min practice period. Perceptual sensitivity scores were determined for each period of watch, and an overall average $d^{\prime}$ was derived from those scores. These data proved remarkably stable. We thus determined our first criterion for establishing task equivalency-a longer rather than shorter session.

Our second criterion applied to outliers. Vigilance tasks typically demonstrate great variability (cf. Koelega, Brinkman, Hendricks, \& Verbaten, 1989). Because our study sought to establish a task of specific detectability for both age groups, we decided a priori to focus on the performance of those participants whose $d^{\prime}$ scores fell in the average range. Those participants whose scores fell two standard deviations below the mean were excluded on the grounds that they did not represent average performance. It is important to note that outliers were excluded during the calibration of the task in Experiment 1, but not in the subsequent experiment.

Furthermore, the $d^{\prime}$ scores also were assessed for the presence of the sensitivity decrement. Studies in adult vigilance, which typically analyze performance over time, often report decreases over time in the efficiency and accuracy of performance (Warm \& Jerison, 1984). Such a worsening of performance has not been reliably demonstrated in research with children (Corkum \& Siegel, 1993; Seidel \& Joschko, 1990). Many researchers of childhood vigilance do not even assess performance across time, choosing instead to report overall performance (e.g., average hits or response latencies). The present study therefore sought to demonstrate the sensi- tivity decrement in all participants and to describe agerelated effects for performance over time.

In addition to the analysis of $d^{\prime}$ scores, we also examined response bias and latency scores. Response bias assesses decision criterion or willingness to respond: $\mathrm{Ob}-$ servers can adopt either a conservative criterion (i.e., become extremely cautious) or a lenient criterion. We utilized the new measure $c$, which offers an estimate of response bias superior to the more traditional $\beta$ (See, Warm, Dember, \& Howe, 1997). Studies of adult vigilance have long evaluated not only sensitivity but also response bias as a means to gain a more complete understanding of attentional changes over time. A response bias increment over the course of a session is typically reported in the adult literature, with observers becoming more conservative with time on task (See et al., 1997). In contrast, both the adult and childhood vigilance literatures have widely applied response latency assessment. Response latencies typically increase over a vigilance session, and this increase has been offered as support for the vigilance decrement (Warm \& Jerison, 1984). Thus, we evaluated whether the task designed would yield the concomitant response bias and response latency increments usually associated with vigilance tasks and whether such changes would appear in both age groups.

\section{Method}

Participants. Thirty-four children ( 7 boys and 27 girls) from the second grade were recruited for the study. Two children identified as outliers were excluded. The remaining 32 children ranged in age from 7 years 4 months to 8 years 8 months, with a mean age of 7 years 11 months. Parents were asked to report any history of attention problems on a sheet sent home with the consent letter. None of the children had received a diagnosis of an attentional problem, nor did the parents indicate any general concerns regarding attention. Thirtyfour adults ( 8 men and 26 women) were recruited from undergraduate psychology courses at Otterbein College. Two participants identified as outliers were excluded. The remaining 32 adults ranged in age from 18 to 46 years (mean age 22). The children were paid $\$ 5$ for their participation, and the college students received course credit.

Procedure. Critical signals and neutral events consisted of blackoutlined white squares presented on a white background. The squares were outlined by $1-\mathrm{mm}$ black lines. Two equal-sized larger squares comprised the neutral event, and two equal-sized smaller squares the critical signal. Stimuli were presented $10 \mathrm{~mm}$ apart at the center of the computer screen. The standard square pair in the neutral event measured $36 \mathrm{~mm}$ for both age groups. The participants sat approximately $15 \mathrm{in}$. from the computer screen. Children performed the task on an iBook laptop (screen size measured $7.5 \times$ 9.5 in.), thus enabling participation in their elementary schools. Adults performed the task on a Macintosh G3 computer (screen size measured $10 \times 13$ in.) in a campus laboratory.

The experimenters tested a variety of stimulus square pairs in order to match to a $d^{\prime}$ in the range of 3.0 to 3.5 , a range defined elsewhere as moderately easy (Craig, 1984). Initial pilot work revealed that a comparison pair comprising squares measuring $26.5 \mathrm{~mm}$ for children and $31.40 \mathrm{~mm}$ for adults might fall within the desired range for a critical signal. Thus, the critical signal and neutral event pairs differed for children by $9.5 \mathrm{~mm}$, but for adults by only $4.6 \mathrm{~mm}$. These stimuli are presented in Figure 1.

These pairs were presented in a 16-min session, comprising a 2min practice sequence followed by a 14-min task. All participants were observed during the practice sequence, and it was determined that they comprehended the task and distinguished between critical 
A)

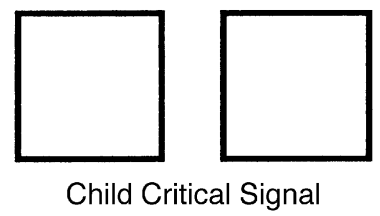

B)

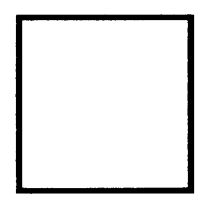

Adult Critical Signal

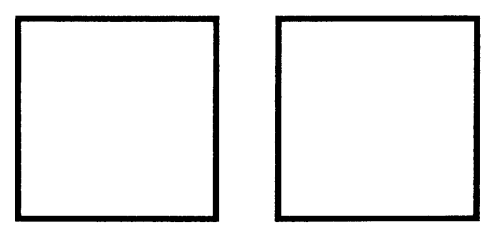

Child Neutral Event

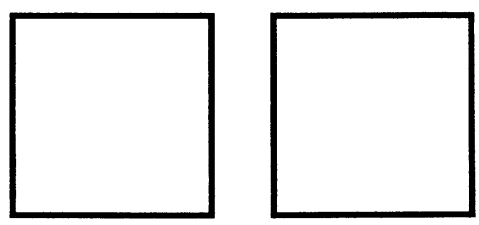

Adult Neutral Event

Figure 1. Critical signals and neutral events for (A) children and (B) adults.

signals and neutral events. All participants performed the task individually in a well-lit environment in which glare was minimal. They were instructed to press a designated key on the computer keyboard only when the critical signal appeared on the screen. Stimuli were presented for $200 \mathrm{msec}$ at a rate of 36 events per minute, with a critical signal probability of .25 . A response to a critical signal was considered a hit if it occurred before the next stimulus was presented. A response to the neutral stimulus was considered a false alarm.

\section{Results}

Means and standard deviations for the $d^{\prime}, c$, and response latency scores are presented in Table 1 for children and adults at each period of watch. Concerning the analyses, perceptual sensitivity scores $\left(d^{\prime}\right)$ were calculated for each participant for each period of watch. Average $d^{\prime}$ scores across the entire vigil were then determined for each of the 32 participants in the child and adult groups. Within each age group-children and adults - the data were separated into two random groups in order to assess the consistency of performance for each age (hereafter we will refer to these divisions as group halves). A 2 (age: child, adult) $\times 2$ (group half: first, second) $\times 7$ (period of watch) split-plot analysis of variance (ANOVA) was conducted on the data. The ANOVA revealed no significant main effect of age $(F<$ 1). Likewise, the main effect of group half was not significant $(F<1)$. Examination of the means for children (3.30 and 3.29 for the first and second halves, respectively) revealed remarkable consistency with means for adults (3.25 and 3.29 for the first and second halves). The main effect of watch was significant $[F(6,360)=$ $2.90, p<.01]$, revealing a sensitivity decrement. All interactions failed to reach significance.

For the remaining analyses, we ran 2 (age) $\times 7$ (period of watch) split-plot ANOVAs. Concerning response bias, the main effect of age was not significant $(F<1)$. The main effect of watch was significant $[F(6,372)=3.87$, $p<.001]$. This main effect was modified by a significant age $\times$ watch interaction $[F(6,372)=2.75, p<.05]$.
Observation of the data presented in Table 1 confirms, as did tests of simple effects, that conservatism levels for children increased across watch periods $[F(6,372)=6.47$, $p<.01]$, whereas conservatism levels for adults remained constant across the entire vigil $(F<1)$.

Response latency scores revealed significant main effects for age and watch $[F(1,62)=71.49, p<.001$, and $F(6,372)=16.37, p<.001$, respectively] and a significant interaction of these variables $[F(6,372)=7.95, p<$ $.001]$. Observation of the data in Table 1 confirms, as did tests of simple effects, that latencies rose over time for children $[F(6,372)=22.97, p<.001]$ but remained relatively stable for adults $(p>.05)$.

\section{Discussion}

Several findings from our calibration study are noteworthy. First, the overall goal of developing a task that was comparable for both children and adults using $d^{\prime}$ matching was successful. The experiment avoided basal and ceiling effects. The absence of an age effect for $d^{\prime}$ suggests that the tasks were equated for both age groups on signal detectability. Finally, the consistency of performance within each age group suggests that sampling error did not occur with our groups.

The significant main effect of watch for $d^{\prime}$ indicates that a short vigilance task can yield a sensitivity decrement in both children and adults. Significant age $\times$ watch interactions for $c$ and response latency indicate that a vigilance decrement was present in children but not in adults; children demonstrated the expected increase in conservatism and response latency over time. Thus, although children and adults showed comparable perceptual sensitivity, adults remained more vigilant. Experiment 2 further explored this potentially important developmental difference.

Having calibrated a task, we next sought to examine the effects of manipulating event rate on performance. Interactions between task parameters and the participant variable of age may be highly informative about the development of attention. Because variations in task parameters 
Table 1

Experiment 1: Perceptual Sensitivity Scores $\left(d^{\prime}\right)$, Response Bias Scores (c) and Response Latency (RT) in All Experimental Conditions

\begin{tabular}{|c|c|c|c|c|c|c|c|c|c|c|c|c|c|c|c|}
\hline \multirow{3}{*}{$\begin{array}{l}\text { Period of } \\
\text { Watch }\end{array}$} & \multicolumn{6}{|c|}{ Children } & \multicolumn{6}{|c|}{ Adults } & \multirow{2}{*}{\multicolumn{3}{|c|}{ Mean }} \\
\hline & \multicolumn{2}{|c|}{$d^{\prime}$} & \multicolumn{2}{|c|}{$c$} & \multicolumn{2}{|c|}{ RT } & \multicolumn{2}{|c|}{$d^{\prime}$} & \multicolumn{2}{|c|}{$c$} & \multicolumn{2}{|c|}{ RT } & & & \\
\hline & $M$ & $S D$ & $M$ & $S D$ & $M$ & $S D$ & $M$ & $S D$ & $M$ & $S D$ & $M$ & $S D$ & $d^{\prime}$ & $c$ & RT \\
\hline 1 & 3.39 & 0.84 & .14 & .32 & 578.30 & 87.77 & 3.48 & 0.62 & .26 & .33 & 484.94 & 73.89 & 3.44 & .20 & 531.62 \\
\hline 2 & 3.33 & 0.66 & .16 & .35 & 616.36 & 77.35 & 3.30 & 0.66 & .28 & .35 & 507 & 72.79 & 3.3 & .22 & 561.91 \\
\hline 3 & 3.39 & 0.69 & .25 & .53 & 636.92 & 97.87 & 3.33 & 0.65 & .32 & .36 & 516.43 & 70.76 & 3.36 & .29 & 576.68 \\
\hline 4 & 3.22 & 0.58 & .34 & .34 & 665.69 & 92.37 & 3.18 & 0.83 & .30 & .38 & 513.56 & 79.48 & 3.20 & .32 & 589.63 \\
\hline 5 & 3.28 & 0.75 & .41 & .34 & 688.92 & 95.70 & 3.21 & 0.79 & .29 & .38 & 516.43 & 79. & 3.25 & .35 & 602.68 \\
\hline 6 & 3.35 & 0.63 & .39 & .34 & 693.91 & 99.15 & 3.29 & 0.82 & .29 & .36 & 510. & 77. & 3.32 & .34 & 602.67 \\
\hline 7 & 3.09 & 0.62 & .49 & .29 & 695.57 & 93.87 & 3.09 & 0.87 & .30 & .45 & 511.24 & 74.54 & 3.09 & .40 & 603.41 \\
\hline Mean & 3.29 & & .31 & & 653.67 & & 3.27 & & .29 & & 508.67 & & & & \\
\hline
\end{tabular}

will influence $d^{\prime}$ values, we did not expect that $d^{\prime}$ values would inevitably remain the same in the subsequent experiment. Indeed, because we planned to have each participant perform two vigils, we anticipated a lower overall $d^{\prime}$. We felt confident, however, that we had devised stimuli that were appropriate for each age group, would allow us to make age-related comparisons, and would avoid basal and ceiling effects.

\section{EXPERIMENT 2}

Despite the significant role of event rate in adult vigilance studies, few studies have examined the role of this parameter in children. Event rate derives from the duration of stimulus presentation and the interstimulus interval. Manipulation of those times adjusts the rate at which targets and neutral events present themselves. Parasuraman and Davies's (1977) taxonomy of vigilance performance among adults identified event rate as one of the most important factors affecting vigilance performance. Typically, as the taxonomy predicts, adult performance efficiency is inversely related to event rate (Warm \& Jerison, 1984).

Interestingly, findings from initial studies in children have contradicted the expectation that performance would suffer under faster event rate conditions. LaurieRose, Bennett-Murphy, Schickedantz, and Tucci (2001), for example, found that young children performed better at faster than at slower event rates. Other reports for children show that performance suffers under slower event rate conditions (Chee, Logan, Schachar, Lindsay, \& Wachsmuth, 1989; van der Meere, Shalev, Borger, \& Gross-Tsur, 1995), whereas optimal performance occurs at relatively fast event rates (Chee et al., 1989). Such studies suggest that a child's performance differs from that of an adult in response to event rate. No direct comparisons, however, have been made between children and adults.

In addition to exploring age-related effects for event rate, this study also sought to replicate the observed sensitivity decrement $\left(d^{\prime}\right)$ across age groups. Finally, we sought to explore further developmental differences in the vigilance decrement ( $c$ and response latency). Through this experiment, we hoped to test the utility of combining an optimal stimulation model and resource models.

\section{Method}

Participants. As in Experiment 1, 32 children (16 boys and 16 girls) from the second grade, ranging in age from 7 years 4 months to 8 years 11 months (mean age 8 years 3 months) were recruited from local elementary schools. Thirty-two adults ( 16 men and 16 women, ranging in age from 18 to 39, mean age 20.75) were recruited from Otterbein College. Recruitment, consent, and payment were identical to the procedures utilized for Experiment 1.

Procedure. Vigilance tasks were designed with a slow (10 events per minute) and a fast (45 events per minute) event rate. The stimuli utilized in these tasks (slow and fast) were identical to those developed in Experiment 1. The probability of a critical signal event was held constant at .3 for both event rate tasks.

The participants completed both 16-min tasks in one sitting. Presentation of the tasks was balanced across age and gender. Each task (slow and fast) consisted of a 2-min practice session followed by seven 2 -min periods of watch. The computers, practice, and task instructions were identical to those in Experiment 1.

\section{Results}

Means and standard deviations for the $d^{\prime}, c$, and response latency scores in the slow and fast event rate conditions for each period of watch are presented in Table 2 for children and adults. Concerning the analyses, a 2 (age: child, adult) $\times 2$ (event rate: slow, fast) $\times 7$ (period of watch) split-plot ANOVA with repeated measures on the latter two variables was performed on $d^{\prime}$ scores. The main effect of age was significant $[F(1,62)=25.42, p<$ $.001]$, with adults $(M=3.08)$ being more sensitive than children $(M=2.37)$ on the tasks. The main effect of event rate was significant $[F(1,62)=27.94, p<.001]$, with the fast event rate $(M=2.92)$ yielding greater perceptual sensitivity than the slow event rate $(M=2.53)$. The age $\times$ event rate interaction approached significance $[F(1,62)=3.72, p=.058]$. Observation of Table 2 reveals that although both groups showed superior performance in the fast event rate condition relative to the slow condition, the adults derived greater benefit. Tests of simple effects revealed the reverse event rate effect was more pronounced for adults $[F(1,62)=26.02, p<.001]$ than for children $[F(1,62)=5.64, p<.05]$.

The main effect of watch was significant $[F(6,372)=$ $3.75, p<.01]$ and was modified by a significant event rate $\times$ watch interaction $[F(6,372)=7.26, p<.001]$. Perusal of the data in Table 2 reveals that sensitivity 


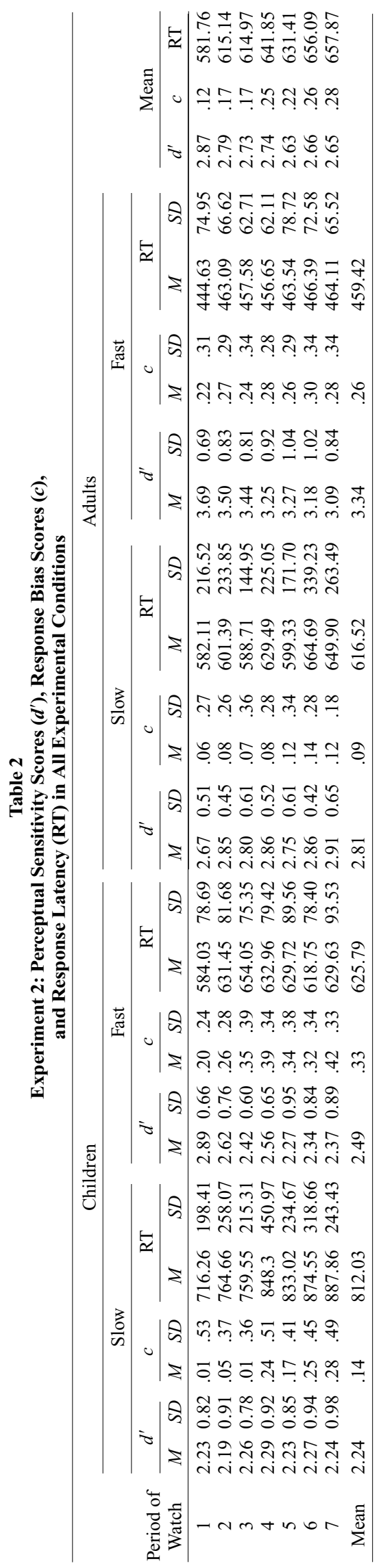

scores were much lower in the slow than in the fast event rate condition. Tests of simple effects revealed that for the slow event rate task, scores remained stable $(F<1)$, whereas for the fast event rate task, scores declined across time $[F(6,372)=11.40, p<.001]$.

To assess the influence of fatigue on performance, a 2 (age) $\times 2$ (event rate) between-factors ANOVA was performed on the last period of watch in the second vigil. The ANOVA revealed a significant main effect of age $[F(1,60)=11.58, p<.01]$, with children displaying less sensitivity $(M=2.19)$ than adults $(2.95)$. The main effect of event rate was also significant $[F(1,60)=4.66$, $p<.05]$, with greater sensitivity apparent in the fast event rate condition $(M=2.82)$ than in the slow event rate condition $(M=2.33)$.

A 2 (age: child, adult) $\times 2$ (event rate: slow, fast) $\times 7$ (period of watch) split-plot ANOVA with repeated measures on the latter two variables was performed on the response bias index $c$. The main effect of event rate was significant $[F(1,62)=29.64, p<.001]$, with the slow event rate condition rendering less conservative scores $(M=.115)$ than the fast event rate condition $(M=.295)$. A significant main effect of watch $[F(6,372)=5.57, p<$ $.001]$ was modified by a significant age $\times$ watch interaction $[F(6,372)=2.09, p=.05]$. Observation of the data in Table 2 reveals, as did tests of simple effects, that adult conservatism remained stable over time $(F<1)$, whereas children's conservatism rose with time on task $[F(6,372)=6.99, p<.001]$.

A parallel ANOVA performed on response latencies to correct detections revealed a significant main effect of age $[F(1,62)=38.62, p<.001]$, with children responding more slowly $(M=718.91)$ than adults $(M=537.97)$. No interactions were observed with age. Significant main effects were observed for both event rate $[F(1,62)=69.23$, $p<.001]$ and period of watch $[F(6,372)=4.86, p<.01]$. These main effects were modified by a significant event rate $\times$ watch interaction $[F(6,372)=3.08, p<.01]$. Tests of simple effects revealed that at each event rate latencies increased significantly over time $[F(6,372)=3.87, p<$ .001 , and $F(6,372)=5.35, p<.001$, respectively, for the slow and fast event rates]. Observation of the data in Table 2 reveals that the increase is both more marked and more variable in the slow event rate condition.

\section{Discussion}

When interpreting the above findings, it is essential to bear in mind that vigilance performance is not a unitary process with a unitary outcome (Warm, 1977). Indeed, the adult literature delineates three types of broad performance outcome, each of which arises from a functionally different mechanism: overall vigilance level, the vigilance decrement, and the sensitivity decrement. As Parasuraman et al. (1989) remarked, the study of these broad performance outcomes informs us about individual differences and, by extension, development. Furthermore, in evaluating the present findings, it is also important to note that interactions between age and measure, 
not main effects, provide the most appropriate indicators of development (McKay et al., 1994).

Overall vigilance performance. The present study reports several significant findings in overall vigilance, or average performance across conditions. First, from a comparative perspective, adults demonstrated higher perceptual sensitivity than did children. All participants, however, showed greater sensitivity in the fast event rate condition. Furthermore, in the fast event rate condition, all participants not only adopted a more conservative response criterion but also exhibited faster response latencies. Whereas this reverse event rate effect has been reported for children (cf. Chee et al., 1989; Laurie-Rose et al., 2001; van der Meere et al., 1995), the finding with adults counters one of the most robust effects reported in the adult vigilance literature - that fast event rate produces a decline in performance. To date, a study of adult vigilance by Posner and Ogden (Posner, 1986) remains, as Warm and Jerison (1984) describe, "the sole experimental demonstration that a fast event rate can facilitate performance in a vigilance task in any way" (p. 28).

We suggest that arousal theory, particularly an optimal stimulation theory that takes into consideration task demands, may help to explain the reverse event rate effect in both groups. The mechanism through which arousal operates might differ, however, for children and adults. Concerning the finding with children, neurobiological data suggest that the early middle childhood years are a time of transition in arousal and regulation of attention (Corbin, Penuel, Bickford, \& Reginald, 1955; Thatcher, 1994). Although 7- and 8-year-olds are qualitatively more similar to adults than are younger children, 7- and 8 -year-olds may have lower levels of arousal than adults. For children with lower levels of arousal and less experience in modulating arousal (Zentall \& Zentall, 1983), faster event rates may optimize stimulation, and thus performance. Furthermore, task parameters may play a role in optimizing performance. If, as Zentall and Zentall have suggested, children are less able inwardly to modulate stimulus-input levels than are adults, they may be more dependent upon task variables to regulate attention. In fact, Ruff (1998) and others have suggested that children, in the process of developing voluntary control of attention, may remain more likely to respond on the basis of potent external cues. High event rate tasks may improve performance in children by providing more instantiations of the target, thereby "capturing" the attention of the child and increasing arousal.

Optimal stimulation theory may also be used to explain our unexpected findings with adults. In the present study, the average $d^{\prime}$ for adults in the fast event rate condition was 3.3, a value described by Craig (1984) as "moderately easy." By contrast, a metaanalytic review by See et al. (1995) reported an average $d^{\prime}$ of 2.43 for adult vigilance tasks, a rating described by Craig as "moderately difficult." A slow event rate task combined with this relatively easier discrimination might have had a "deenergizing" effect, and the concomitant lower levels of arousal might have impeded performance. Indeed, See et al. (1995) suggested that much remains to be known about vigilance tasks employing slower event rates.

From a developmental perspective, the age $\times$ event rate interaction suggests that adult performance improved to a greater extent in the fast event rate condition than did the performance of children. Scores were $16 \%$ better for adults in the fast event rate condition, whereas children gained only a $10 \%$ advantage. In the case of children, therefore, task parameter changes beyond event rate manipulations might be required in order to increase arousal and improve performance. Vigilance tasks, even fast ones, remain relatively dull. These data call for clarification of the conditions under which the reverse event rate effect appears in children and adults and for examination of factors that might influence arousal levels.

Vigilance decrement. The second performance outcome, the vigilance decrement, points specifically to a change in detection rate, typically resulting from a decline in responsiveness to all stimuli across a session. Such a decline reflects a shift in criterion over the course of the vigil from lenient to conservative. A concomitant rise in response latency typically accompanies that criterion shift (Parasuraman, 1984). Regarding the typical shift in criterion, children demonstrated the expected vigilance decrement. As evidenced by the age $\times$ watch interaction, children showed increasing conservatism over time. However, across both groups, response latency scores clearly indicated a vigilance decrement. Reaction times increased across periods of watch for both children and adults at both event rates. That increase became more pronounced in the slow event rate condition, another indication of a reverse event rate effect. Across the seven periods of watch, average latency for both groups increased by a mean of $119 \mathrm{msec}$ in the slow event rate condition, as compared with $32 \mathrm{msec}$ in the fast condition.

Cooley and Morris (1990) suggested that optimal stimulation theory can account for performance changes over time. According to Parasuraman (1984), lowered arousal is clearly a concomitant of the vigilance decrement, although arousal level alone cannot adequately account for that decrement. If, as Matthews and Davies (1998a) and van der Meere and colleagues (e.g., van der Meere \& Stemerdink, 1999) have argued, resource availability expands under conditions of sufficiently high task demand, it would follow that less demanding vigilance tasks would lead to a loss of energetic arousal over time (Matthews \& Davies, 1998b). This lowered energetic arousal would likely affect effort (Matthews \& Davies, 1998b).

In the present study, children became more conservative over time. Although the trend failed to reach significance, adults' performance also showed increased conservatism over time. The expected rise in conservatism in children's performance might reflect a decline in their arousal. For adults, however, the specific combination of task parameters - a short vigil length and high signal de- 
tectability - might explain the absence of a significant conservative criterion increment. Other studies using a shortened vigil in adults have failed to document the typical rise in conservatism (cf. Nuechterlein, Parasuraman, $\&$ Jiang, 1983). In our experiment, a combination of several task parameters, including easier task detectability, could have maintained stable arousal and performance levels for adults.

This developmental finding might indicate that the child's optimal stimulation is not only narrower in range than that of adults (Zentall \& Zentall, 1983) but also shorter in duration. Clearly, future studies must explore how individual differences in optimal stimulation vary with time on task. Explaining developmental differences in decrements and performance over time is necessary for the theory to be fully effective.

Sensitivity decrement. The sensitivity decrement refers to a decline in $d^{\prime}$ scores over the course of a session. In lieu of arousal and optimal stimulation models, resource allocation models are typically offered as an explanation for the sensitivity decrement (Parasuraman, 1984). These models assume that capacity limitations exist in information processing and that various task parameters may differentially exhaust available resources. High event rate tasks or tasks that impose greater burdens stretch available resources to their limits (Fisk \& Schneider, 1981).

In the present study, a sensitivity decrement was observed for both groups (main effect of watch), and the decrement was most marked in the fast event rate condition. We observed no developmental differences in sensitivity. Thus, one could conclude that although the high demands of the fast event rate condition tax resources and lead to a sensitivity decrement, the process by which children and adults allocate resources is similar. In other words, the absence of a developmental effect suggests that 7 - and 8-year-old children and adults possess qualitatively similar abilities to perceive critical signals and allocate attention to that perceptual process over time, at least under these conditions. However, one must exercise caution in drawing such a conclusion from this study. Although there may be no developmental differences in allocation, it is also possible that the type of task we used did not require much in the way of allocation (Matthews \& Davies, 1998a). The findings warrant further study.

\section{GENERAL DISCUSSION}

Seeking to reduce the distance between child and adult vigilance studies, we applied a procedure used primarily in the psychophysical literature to equate vigilance tasks across age. First, we demonstrated that tasks can be equated across groups. In the first experiment, we equated tasks on signal detectability so as to avoid basal and ceiling effects in the second experiment. Sufficient variability existed in the data from Experiment 2 to indicate that the task was neither too easy for adults nor too hard for children.
Second, these equated tasks produced typical vigilance findings. Both experiments evidenced the typical decline in performance over time. The sensitivity decrement emerged from the fast event rate condition, with a similar pattern of decline-17\% in Experiment 2-for both children and adults. That pattern closely approximates the 20\% value that Swets (1977) described as the typical magnitude of sensitivity decline in vigilance studies. Interestingly, Swets based this value on studies using longer vigilance sessions. Finally, the increase in response latency across periods of watch confirmed the vigilance decrement. Our findings thus indicated that vigilance tasks of equally short lengths can be used for both children and adults. They support previous research in which sensitivity and vigilance decrements were obtained in abbreviated vigils with adults (Laurie-Rose, Bennett-Murphy, Byard, \& Nikzad, 2002; Nuechterlein et al., 1983; Temple et al., 1997).

Third, because we achieved task equivalency, we were able to identify subtle developmental differences arising from task parameter manipulations. As anticipated, $d^{\prime}$ in the second experiment varied from that of the first experiment because of changes in task parameters and the demands of performing a second vigil. Statistical analyses revealed that the load imposed by performing a second vigil produced more deleterious effects in children than in adults. Interestingly, the nature of the changes in $d^{\prime}$ from Experiment 1 to Experiment 2 helped lift to the surface subtle differences between adults and children. These changes across the two experiments might suggest that group differences exist in response to parametric changes. Thus, whereas children in this study appeared more vulnerable to the demands of a second vigil and more likely to evidence a vigilance decrement, adults showed greater change in performance as a result of the event rate manipulation. In a finding of crucial importance from the developmental perspective, the data revealed the presence of the reverse event rate effect in both children and adults.

Finally, our findings support the utility of combining optimal stimulation theory with the resource allocation approach. Such an approach falls in line with experts in both the adult literature (e.g., Matthews \& Davies, 1998a) and the clinical child literature (cf. van der Meere et al., 1992). Furthermore, the findings highlight developmental differences in arousal and the modulation of attention. Although the data suggest interesting developmental differences, we propose these developmental explanations cautiously, recognizing that our conclusions must be limited to 7- and 8-year-old children. Future research that employs such a technique with several age groups will be able to draw more specific conclusions regarding development.

In his now classic work on control by equation in comparative psychology, Bitterman (1965, p. 400) stated that one could not know how to produce "a set of conditions which ... will make sensory and motor demands exactly equal" for two highly different subject groups-in his 
case, two different animal species. Yet, he continued, "meaningful comparisons are still possible, because for control by equation we may substitute what I call control by systematic variation" (p. 400). We propose that, by equating for $d^{\prime}$ in our first experiment, we achieved such meaningful comparisons in our subsequent experiment. Thus, our method allowed us to proceed farther than similar work has gone in studying the attentional processes of children and in comparing those processes to those of adults. Direct comparisons between children and adults, as well as examination of the interaction between age and task variables, will promote a more comprehensive view of the development of sustained attention. The present study thus helps demonstrate the importance of utilizing $d^{\prime}$ measures in building new methodology and theory in the field of attention.

\section{REFERENCES}

BeLl, M. A. (1998). Frontal lobe function during infancy: Implications for the development of cognition and attention. In J. E. Richards (Ed.), Cognitive neuroscience of attention: A developmental perspective (pp. 287-316). Mahwah, NJ: Erlbaum.

Berch, D. B., \& Kanter, D. R. (1984). Individual differences. In J. S. Warm (Ed.), Sustained attention in human performance (pp. 143178). Chichester, U.K.: Wiley.

Berlyne, D. E. (1960). Conflict, arousal, and curiosity. New York: McGraw-Hill.

Bitterman, M. E. (1965). Phyletic differences in learning. American Psychologist, 20, 396-410.

BORNSTEIN, M. H. (1990). Attention in infancy and the prediction of cognitive capacities in childhood. In J. T. Enns (Ed.), The development of attention: Research and theory (pp. 3-19). Amsterdam: Elsevier, North-Holland.

Chee, P., Logan, G., Schachar, R., Lindsay, P., \& Wachsmuth, R. (1989). Effects of event rate and display time on sustained attention in hyperactive, normal, and control children. Journal of Abnormal Child Psychology, 17, 371-391.

COOLEY, E. L., \& MoRRIS, R. D. (1990). Attention in children: A neuropsychologically based model for assessment. Developmental Neuropsychology, 6, 239-274.

Corbin, H., Penuel, F., Bickford, R. G., \& Reginald, G. (1955). Studies of the electroencephalogram of normal children: Comparison of visual and automatic frequency analyses. Electroencephalogram in Clinical Neurophysiology, 7, 15-28.

CORKUM, P. V., \& SIEGEL, L. S. (1993). Is the continuous performance task a valuable research tool for use with children with attentiondeficit-hyperactivity disorder? Journal of Child Psychology \& Psychiatry \& Allied Disciplines, 34, 1217-1239.

CRAIG, A. (1984). Human engineering: The control of vigilance. In J. S. Warm (Ed.), Sustained attention in human performance (pp. 247291). Chichester, U.K.: Wiley.

Davies, D. R., Jones, D. M., \& TAYlor, A. (1984). Selective- and sustained-attention tasks: Individual and group differences. In R. Parasuraman \& D. R. Davies (Eds.), Varieties of attention (pp. 395447). Orlando, FL: Academic Press.

DeWolfe, N. A., Byrne, J. M., \& Bawden, H. N. (1999). Early clinical assessment of attention. Clinical Neuropsychologist, 13, 458-473.

Enns, J. T., Brodeur, D. A., \& TRICK, L. M. (1998). Selective attention over the lifespan: Behavioral measures. In J. E. Richards (Ed.), Cognitive neuroscience of attention: A developmental perspective (pp. 393-418). Mahwah, NJ: Erlbaum.

FisK, A. D., \& SCHNEIDER, W. (1981). Control and automatic processing during tasks requiring sustained attention: A new approach to vigilance. Human Factors, 29, 653-660.

Giambra, L. M., \& Quilter, R. E. (1988). Sustained attention in adult- hood: A unique, large sample, longitudinal and multicohort analysis using the Mackworth clock-test. Psychology \& Aging, 3, 75-83.

GoldSMith, D. F., \& RogofF, B. (1997). Mothers' and toddlers' coordinated joint focus of attention: Variations with maternal dysphoric symptoms. Developmental Psychology, 33, 113-119.

GreenberG, L. M., \& WaldMan, I. D. (1993). Developmental normative data on the test of variables of attention (T.O.V.A.). Journal of Child Psychology \& Psychiatry, 34, 1019-1030.

HALPERIN, J. M. (1996). Conceptualizing, describing, and measuring components of attention. In G. R. Lyon \& N. A. Krasnegor (Eds.), Attention, memory and executive function (pp. 119-136). Baltimore: Paul H. Brookes.

Halperin, J. M., Sharma, V., Greenblatt, E., \& Schwartz, S. T. (1991). Assessment of the continuous performance test: Reliability and validity in a nonreferred sample. Psychological Assessment: A Journal of Consulting \& Clinical Psychology, 3, 603-608.

Hatfield, J. L., \& Loeb, M. (1968). Sense mode and coupling in a vigilance task. Perception \& Psychophysics, 4, 29-36.

HatField, J. L., \& SoderQuist, D. R. (1970). Coupling effects and performance in vigilance tasks. Human Factors, 12, 351-359.

Hockey, G. R. J. (1984). Varieties of attentional states. In R. Parasuraman \& D. R. Davies (Eds.), Varieties of attention (pp. 449-483). Orlando, FL: Academic Press.

Koelega, H. S., Brinkman, J. A., Hendriks, L., \& Verbaten, M. N. (1989). Processing demands, effort, and individual differences in four different vigilance tasks. Human Factors, 31, 45-62.

Laurie-Rose, C., Bennett-MurPhy, L., Byard, L., \& Nikzad, K. (2002). The role of the big-five personality factors in vigilance performance and workload. European Journal of Personality, 16, 185-200.

Laurie-Rose, C., Bennett-Murphy, L., Schickedantz, B., \& TucCI, J. (2001). The effects of event rate and signal probability on children's vigilance. Journal of Clinical \& Experimental Neuropsychology, 23, 215-224.

LeUng, J. P., LEUNG, P. W. L., \& TANG, C. S. K. (2000). A vigilance study of event rate and extratask stimulation. Journal of Developmental \& Physical Disabilities, 12, 187-201.

LEVY, F. (1980). The development of sustained attention (vigilance) and inhibition in children: Some normative data. Journal of Child Psychology \& Psychiatry, 21, 77-84.

Losier, B. J., McGrath, P. J., \& Klein, R. M. (1996). Error patterns on the continuous performance test in nonmedicated and medicated samples of children with and without ADHD: A meta-analytic review. Journal of Child Psychology \& Psychiatry \& Allied Sciences, 37, 971-987.

Macmillan, N., \& Creelman, C. D. (1991). Detection theory: A user's guide. New York: Cambridge University Press.

Matthews, G., \& Davies, D. R. (1998a). Arousal and vigilance. In R. R. Hoffman, M. F. Sherrick, \& J. S. Warm (Eds.), Viewing psychology as a whole: The integrative science of William N. Dember (pp. 113-144). Washington, DC: American Psychological Association.

Matthews, G., \& Davies, D. R. (1998b). Arousal and vigilance: Still vital at fifty. Proceedings of the Human Factors \& Ergonomics Society, 42, 772-776.

McKaY, K., Halperin, J. M., Schwartz, S. T., \& Sharma, V. (1994). Developmental analysis of three aspects of information processing: Sustained attention, selective attention, and response organization. Developmental Neuropsychology, 10, 121-132.

NICHD Early Child CaRe Research Network (2003). Do children's attention processes mediate the link between family predictors and school readiness? Developmental Psychology, 39, 581-593.

Nuechterlein, K. H., Parasuraman, R., \& Jiang, Q. (1983). Visual sustained attention: Image degradation produces rapid sensitivity decrement over time. Science, 220, 327-329.

PaRASURAMAN, R. (1984). Sustaine attention in detection and discrimination. In R. Parasuraman \& D. R. Davies (Eds.), Varieties of attention (pp. 243-271). Orlando, FL: Academic Press.

Parasuraman, R., \& Davies, D. R. (1977). A taxonomic analysis of vigilance performance. In R. R. Mackie (Ed.), Vigilance: Theory, operational performance, and physiological correlates (pp. 559-574). New York: Plenum. 
Parasuraman, R., \& Mouloua, M. (1987). Interaction of signal discriminability and task type in vigilance decrement. Perception \& Psychophysics, 41, 17-22.

Parasuraman, R., Nestor, P., \& Greenwood, P. (1989). Sustainedattention capacity in young and older adults. Psychology \& Aging, 4, 339-345.

Posner, M. I. (1986). Chronometric explorations of mind. New York: Oxford University Press.

Rebok, G. W., Smith, C. B., Pascualvaca, D. M., Mirsky, A. F., AnTHONY, B. J., \& Kellam, S. G. (1997). Developmental changes in attentional performance in urban children from 8 to 13 years. Child Neuropsychology, 3, 28-46.

RUFF, H. A. (1998). Summary and commentary: Selective attention. Its measurement in a developmental framework. In J. E. Richards (Ed.), Cognitive neuroscience of attention: A developmental perspective (pp. 419-426). Mahwah, NJ: Erlbaum.

See, J. E., Howe, S. R., Warm, J. S., \& Dember, W. N. (1995). Metaanalysis of the sensitivity decrement in vigilance. Psychological Bulletin, 117, 230-249.

SeE, J. E., WARM, J. S., Dember, W. N., \& Howe, S. R. (1997). Vigilance and signal detection theory: An empirical evaluation of five measures of response bias. Human Factors, 39, 14-29.

SEIDEL, W. T., \& JoschKo, M. (1990). Evidence of difficulties in sustained attention in children with ADHD. Journal of Abnormal Child Psychology, 18, 217-229.

SwETS, J. (1977). Signal detection theory applied to vigilance. In R. R. Mackie (Ed.), Vigilance: Theory, operational performance, and physiological correlates (pp. 705-718). New York: Plenum.

Sykes, D. H., Douglas, V. I., Weiss, G., \& Minde, K. K. (1971). Attention in hyperactive children and the effect of Ritalin. Journal of Child Psychology \& Psychiatry, 12, 129-139.

TeICHNer, W. H. (1974). The detection of a simple visual signal as a function of time of watch. Human Factors, 16, 339-353.

Temple, J. G., Warm, J. S., Dember, W. N., Jones, K. S., LaGrange, C. M., \& Matthews, G. (1997). The effects of caffeine and signal salience on performance, workload, and stress in an abbreviated vigilance task. Human Factors, 42, 183-194.

ThatChER, R. (1994). Cyclic cortical reorganization: Origins of human cognitive development. In G. Dawson and K. W. Fischer (Eds.),
Human behavior and the developing brain (pp. 232-268). New York: Guilford Press.

Tomporowski, P. D., \& Tinsley, V. F. (1996). Effects of memory demand and motivation on sustained attention in young and older adults. American Journal of Psychology, 109, 187-204.

Trommer, B. L., Hoeppner, J. B., Lorber, R., \& Armstrong, K. (1988). Pitfalls in the use of a continuous performance test as a diagnostic tool in attention deficit disorder. Developmental \& Behavioral Pediatrics, 9, 339-345.

TyLer, D. M., WAAG, W. L., \& Halcomb, C. G. (1972). Monitoring performance across sense modes: An individual differences approach. Human Factors, 14, 539-547.

VAN DER MEere, J., SHAlev, R., Borger, N., \& Gross-Tsur, V. (1995). Sustained attention, activation, and MPH in ADHD: A research note. Journal of Child Psychology \& Psychiatry \& Allied Disciplines, $\mathbf{3 6}_{2}$ 697-703.

van der Meere, J., \& Stemerdink, N. (1999). The development of state regulation in normal children: An indirect comparison with children with ADHD. Developmental Neuropsychology, 16, 213-225.

van der Meere, J., VReeling, H. J., \& Sergeant, J. J. (1992). A motor presetting experiment in hyperactive, learning disabled and control children. Journal of Child Psychology \& Psychiatry \& Allied Disciplines, 33, 1347-1354.

WARM, J. S. (1977). Psychological processes in sustained attention. In R. R. Mackie (Ed.), Vigilance: Theory, operational performance, and physiological correlates (pp. 623-644). New York: Plenum.

WARM, J. S., \& DEMBER, W. N. (1998). Tests of vigilance taxonomy. In R. R. Hoffman, M. F. Sherrick, \& J. S. Warm (Eds.), Viewing psychology as a whole: The integrative science of William N. Dember (pp. 87-112). Washington, DC: American Psychological Association. WARM, J. S., \& JERISON, H. J. (1984). The psychophysics of vigilance. In J. S. Warm (Ed.), Sustained attention in human performance (pp. 15-59). Chichester, U.K.: Wiley.

Zentall, S. S., \& Zentall, T. R. (1983). Optimal stimulation: A model of disordered activity and performance in normal and deviant children. Psychological Bulletin, 94, 446-471.

(Manuscript received August 21, 2003; revision accepted for publication May 13, 2004.) 